\title{
Search for Single Top Production with CDF
}

\author{
T. Kikuchi \\ University of Tsukuba \\ Tsukuba, Ibaraki 315, Japan \\ S. K. Wolinski \\ Physics Department, University of Michigan, \\ Ann Arbor, Michigan 48109, U.S.A. \\ L. Demortier \\ Physics Department, Rockefeller University \\ New York, 10021, U.S.A. \\ S. Kim \\ University of Tsukube \\ Tsukuba, Ibaraki 315, Japan \\ P. Savard \\ Physics Department, University of Toronto, \\ Toronto, Ontario M5S 1A7, Canada
}

(For The CDF Collaboration)

\begin{abstract}
We search for Standard Model single-top-quark production in the $W$-gluon fusion and $W^{\star}$ channels using $106 \mathrm{pb}^{-1}$ of data from $p \bar{p}$ collisions at $\sqrt{s}=1.8 \mathrm{TeV}$ collected with the Collider Detector at Fermilab. We set an upper limit at 95\% C.L. on the combined $W g+W^{*}$ single-top cross section of $13.5 \mathrm{pb}$, roughly five-and-a-half times larger than the standard model prediction. Separate $95 \%$ C.L. upper limits in the $W g$-fusion and $W^{\star}$ channels are also determined and are found to be 13.5 and $12.9 \mathrm{pb}$ respectively.
\end{abstract}

\section{Introduction}

The observation of the top quark in $p \bar{p}$ collisions at the Fermilab Tevatron has relied on strong pair production, typically $q \bar{q} \rightarrow g \rightarrow t \bar{t}$. A top quark can also be produced singly, in association with a $\mathrm{b}$ quark, through the electroweak interaction. Two single-top processes, "W-gluon fusion" $\left(q g \rightarrow t b q^{\prime}\right)$ and " $W^{\star "}\left(q q^{\prime} \rightarrow t b\right)$, have predicted cross sections of $1.70 \mathrm{pb}^{1}$ and $0.73 \mathrm{pb}^{2}$, respectively, compared to $t \bar{t}$ pair production at $5.1 \mathrm{pb}^{3,4}$. We report on a search and limit on the two single-top processes, both separately and combined. 


\section{Event Selection}

In this search, we use $106 \mathrm{pb}^{-1}$ of data from $p \bar{p}$ collisions at $\sqrt{s}=1.8 \mathrm{TeV}$ collected with the Collider Detector at Fermilab ${ }^{5}$. In the Standard Model, the final state of a single-top event consists of $W$ decay products plus two or three jets ${ }^{\mathrm{a}}$ : one $b$-quark jet from the top decay, a second $b$-quark jet from the $t W b$ vertex in $W^{\star}$ events and from initial state gluon splitting in $W$-gluon fusion events, and, in the case of $W$-gluon fusion only, a third jet from the recoiling light quark.

We give below a summary of our event selections (more details can be found elsewhere $\left.{ }^{6}\right)$. We restrict our single-top search to events with evidence of a leptonic $W$ decay: an isolated ${ }^{7}$ electron (muon) candidate with $E_{T}\left(P_{T}\right)>20 \mathrm{GeV}(\mathrm{GeV} / \mathrm{c})$ and $E_{T}>20 \mathrm{GeV}$ from the neutrino. Jets are reconstructed using a cone of fixed radius $\Delta R \equiv \sqrt{\Delta \eta^{2}+\Delta \phi^{2}}=0.4$. Events are required to have one, two, or three jets with $E_{T}>15 \mathrm{GeV}$ and $|\eta|<2.0$; at least one jet must be identified as likely to contain a $b$-quark (" $b$-tagged") using displaced-vertex information from the Silicon Vertex Detector $(\mathrm{SVX})^{8}$.

\section{Backgrounds}

The dominant backgrounds consist of QCD multijet processes (mainly $W+$ jets) and $t \bar{t}$ production ${ }^{6}$. The largest component of the non- $t \bar{t}$ background in the SVX-tagged $W+$ jets sample is inclusive $W$ production in association with heavy-flavor jets (e.g. $p \bar{p} \rightarrow W g$, followed by $g \rightarrow b \bar{b})$. Additional sources include "mistags", in which a light-quark jet is erroneously identified as heavy-flavor, "non- $W$ " (e.g. direct $b \bar{b}$ production), and smaller contributions from $W W, W Z$, and $Z$ +heavy-flavor. The mistag and non- $W$ rates are estimated from data, the $W$ theavy-flavor rates from Monte Carlo normalized to data, and the smaller sources such as diboson production from Monte Carlo normalized to theory predictions ${ }^{9}$.

\section{Combined Measurement}

To measure the combined $W g$-fusion $+W^{\star}$ single-top cross section, we use a kinematic variable that is virtually identical for the two single-top processes and yet is separated from the backgrounds: the scalar sum $H_{T}$ of the transverse energies of the lepton, $\not_{T}$ and all jets in the event. The signal significance is improved by requiring the top-quark mass reconstructed from the lepton, neutrino, and leading $b$-tagged jet momenta to lie within a window of $35 \mathrm{GeV} / c^{2}$ of the nominal top mass of $175 \mathrm{GeV} / c^{2}$. Our background predictions are given in Table 1. For our $t \bar{t}$ prediction, we use the theoretical prediction mentioned above ${ }^{4}$. We observe 65 events in the data.

We perform a maximum-likelihood fit of the data distribution of $H_{T}$ to a linear superposition of Monte Carlo distributions for single-top signal, $t \bar{t}$ background, and non-top background. We model the shape of the $H_{T}$ distribution for all sources of non-top background with VECBOS ${ }^{10}$-generated $W b \bar{b}$ events. VECBOS adequately

${ }^{\mathrm{a}}$ Gluon radiation can increase this number. 
reproduces the $H_{T}$ and $M_{\ell \nu b}$ distributions for the $b$-tagged $W+1$ jet data before the $M_{\ell \nu b}$ cut, a sample in which the top content is expected to be very small. The observed $H_{T}$ distribution agrees ${ }^{\text {a }}$ with the spectrum derived from Monte Carlo calculations when the latter are normalized to the a priori predicted numbers of events.

Table 1. Predicted numbers of signal and background events for the combined analysis.

\begin{tabular}{lcccc}
\hline Process & $W+1$ jet & $W+2$ jet & $W+3$ jet & $W+1,2,3$ jet \\
\hline$W g$ & 0.80 & 1.50 & 0.71 & $3.00 \pm 0.63$ \\
$W^{\star}$ & 0.25 & 0.80 & 0.23 & $1.28 \pm 0.27$ \\
$t \bar{t}$ & 0.21 & 2.28 & 5.91 & $8.40 \pm 2.7$ \\
non-top & 37.4 & 13.9 & 2.7 & $54.0 \pm 11.0$ \\
\hline
\end{tabular}

We set an upper limit on the cross section using the likelihood function:

$$
\mathcal{L}\left(\beta_{s}, \beta_{t \bar{t}}, \beta_{n t}\right)=G_{1}\left(\beta_{t \bar{t}}\right) \times G_{2}\left(\beta_{n t}\right) \times \mathcal{L}_{\text {shape }}\left(\beta_{s}, \beta_{t \bar{t}}, \beta_{n t}\right) .
$$

where $\beta_{s}, \beta_{t \bar{t}}$ and $\beta_{n t}$ are fit parameters representing, respectively, the fractions of the predicted numbers of single-top, $t \bar{t}$ and non-top events that are needed to fit the data. The functions $G_{1}$ and $G_{2}$ are Gaussian likelihoods constraining the background fractions $\beta_{t \bar{t}}$ and $\beta_{n t}$ to their expected values, and $\mathcal{L}_{\text {shape }}$ represents the joint probability density for observing all the data events at their respective values of $H_{T}$.

To extract upper limits on the single-top production rate, we construct a probability distribution $f\left(\beta_{s}\right)$ by maximizing $\mathcal{L}\left(\beta_{s}, \beta_{t \bar{t}}, \beta_{n t}\right)$, for each value of $\beta_{s}$, with respect to $\beta_{t \bar{t}}$ and $\beta_{n t}$, and multiplying the result with a flat prior distribution for $\beta_{s}$. We then convolute $f\left(\beta_{s}\right)$ with a Gaussian smearing function whose width equals the sum in quadrature of contributions from all sources of systematic uncertainty ${ }^{11}$. Each contribution is of the form $\sqrt{(\beta \Delta A)^{2}+(\Delta S)^{2}}$, where $\Delta A$ is the effect of the systematic uncertainty on the signal acceptance, $\Delta S$ its effect on the shape of the $H_{T}$ distribution, and $\beta$ the integration variable in the convolution. We obtain $17 \%$ for $\Delta A$ and 0.33 events for $\Delta S$, the dominant uncertainties being jet energy scale, and initial and final state gluon radiation. Finally, the smeared distribution is integrated to find the $95 \%$ confidence level upper limit on single-top production. We calculate this limit to be $\beta_{95}=5.57$, corresponding to a cross section of $13.5 \mathrm{pb}$.

\section{Individual Channel Measurments}

For the individual measurements, we select events with exactly two jets. Also, the $t \bar{t}$ production cross section measured by $\mathrm{CDF}^{9}$ is used instead of the theoretical value. Apart from these differences, the maximum likelihood fit, the calculation of the systematic uncertainties, and the determination of the $95 \%$ confidence limit is done according to the procedure described in the combined analysis.

a A Kolmogorov-Smirnov test yields a confidence level of $50 \%$. 
Further enhancement of the $W$-gluon fusion component in the single-tag sample can be obtained by considering that the light-quark jet in $W$-gluon fusion events is about twice as likely to be in the same hemisphere as the outgoing (anti)proton beam when a (anti)top quark is produced. Thus the product $Q \times \eta$ of the primary lepton charge and the untagged jet pseudorapidity has a strongly asymmetric distribution. For this measurement, we require exactly one $b$-tagged jet in the event. The signal significance is improved by requiring the top-quark mass reconstructed from the lepton, neutrino, and the $b$-tagged jet momenta to lie in the window $145 \leq M_{\ell \nu b} \leq$ $205 \mathrm{GeV} / c^{2}$. The predicted number of signal and background events are $1.4 \pm 0.3$ and $13.0 \pm 2.2$, respectively. Following the definitions above, we obtain $19 \%$ for $\Delta A$ and 0.16 events for $\Delta S$. We observe 15 events in the data and set a $95 \%$ limit of $13.5 \mathrm{pb}$.

For our $W^{*}$ analysis, we fit the reconstructed top quark mass and require at least one $b$-tagged jet. In this case, since both jets can be $b$-tagged, the top mass is reconstructed from the momenta of the primary lepton, the neutrino, and the jet with the largest $\eta(-\eta)$ when a top (antitop) is produced. This assignment of a jet to the $b$-quark from top decay is correct $64 \%$ of the time. The predicted number of signal and background events are $1.2 \pm 0.2$ and $31.5 \pm 4.7$, respectively. For this analysis, the value of $\Delta A$ is $15 \%$ and the value of $\Delta S$ is 0.87 events. We observe 42 events in the data and set a $95 \%$ limit of $12.9 \mathrm{pb}$.

\section{Conclusion}

We have searched for Standard Model single-top-quark production. No significant excess above expected backgrounds was observed. We then set an upper limit at $95 \%$ C.L. on the combined $W g+W^{\star}$ single-top cross section of $13.5 \mathrm{pb}$, roughly five-and-a-half times larger than the standard model prediction. Separate $95 \%$ C.L. upper limits in the $W$-gluon fusion and $W^{\star}$ channels were also determined and were found to be 13.5 and $12.9 \mathrm{pb}$, respectively.

\section{References}

1. T. Stelzer, Z. Sullivan, and S. Willenbrock, Phys. Rev. D 56, 5919 (1997). All cross sections we quote are for a top quark mass of $175 \mathrm{GeV} / c^{2}$.

2. M. Smith and S. Willenbrock, Phys. Rev. D 54, 6696 (1996).

3. S. Willenbrock and D. Dicus, Phys. Rev. D 34, 155 (1986); S. Dawson and S. Willenbrock, Nucl. Phys. B 284, 449 (1987); C.-P. Yuan, Phys. Rev. D 41, 42 (1990); R. Ellis and S. Parke, Phys. Rev. D 46,3785 (1992); D. Carlson and C.-P. Yuan, Phys. Lett. B306, 386 (1993).

4. R. Bonciani et al, Nucl. Phys. B 529, 424-450 (1998).

5. F. Abe et al., Nucl. Instr. Meth. Phys. Res. A 271, 387 (1988); D. Amidei et al., ibid., 350, 73 (1994); P. Azzi et al., ibid., 360, 137 (1995).

6. F. Abe et al., Phys. Rev. Lett. 79, 3819 (1997).

7. A lepton is "isolated" if the non-lepton $E_{T}$ in an $\eta-\phi$ cone of 0.4 centered on the lepton is less than $10 \%$ of the lepton's $E_{T}$ or $P_{T}$.

8. F. Abe et al., Phys. Rev. Lett. 74, 2626 (1995). 
9. F. Abe et al., Phys. Rev. Lett. 80, 2773 (1998).

10. F.A. Berends, W.T. Giele, H. Kujif and B. Tausk, Nucl. Phys. B 357, 32 (1991).

11. The treatment of systematic uncertainties follows F. Abe et al., PRD 43:664-686, 1991. 